\title{
Frequency-effective command and measurement system of spacecraft
}

\author{
V. Evstratko \\ Institute of engineering physic and radio electronics, \\ Siberian federal university \\ Krasnoyarsk, Russian Federation \\ e-mail: evstrafly@list.ru
}

\author{
I. Tikhonenko \\ Institute of engineering physic and radio electronics, \\ Siberian federal university \\ Krasnoyarsk, Russian Federation \\ e-mail: silencebob@yandex.ru
}

\begin{abstract}
This paper provides description of frequencyeffective command and measurement system. The rise of frequency resource usage efficiency is achieved by means of application of simple digital modulation for simultaneous range measurement and command and telemetry data transmission.
\end{abstract}

Keywords - ranging, telemetry, telecomand, PM, PSK, frequency efficiency, CCSDS.

\section{INTRODUCTION}

Spacecraft command and measurement system (SC CMS) is intended for spacecraft and its functional subsystems control at all SC operating stages. SC operating modes and functions management is performed by transmission of telecommands (TC) via radiochannel from ground control station (GCS). Telemetry data (TM) of units and subsystems status and functioning are transferred via return channel. Beyond that command and measurement system provides SC current navigational parameters measurement, where two of basic parameters are SC range (RANGING) and speed in relation to SCC [3].

\section{StRUCTURE DeSCRIPTION}

There are several types of SC CMS for the moment which are differentiated by design of TC transferring radiolink, design of TM radiolink and RANGING radiolink. [1].

Fig. 1 shows SC CMS block diagram recommended by
CCSDS (Consultative Committee for Space Data Systems) standard [1].

SC CMS, which diagram is shown on fig. 1, is designed for transmission of TC data from ground control complex (GCS) to SC; telemetry data transmission from SC to GCS; SC-GCS range measurement (further RANGING).

Scheme operates in the following way. TC data enter the input 1 of TC modulator, TC subcarrier frequency enter the modulator input 2. From modulator output 3 modulated signal on subcarrier frequency enters one of the adder inputs. RANGING signal enters the other summarizer input. Signal from the summarizer output, which is represented as a sum of TC and RANGING signals, enters the first input of analog phase modulator, uplink carrier frequency enters the second input of phase modulator. Further wave modulated with carrier frequency enters the GCS transmitting antenna, and then enters the $\mathrm{SC}$ receiving antenna.

On SC board signal from receiving antenna enters the analog phase demodulator input 1. Signal from demodulator output enters the inputs of two filters: RANGING signal filter and TC signal filter. TC signal, extracted by the means of matched filter, enters the first input of TC demodulator, telecommand data from the second output of demodulator enter the SC hardware. RANGING signal, extracted by the instrumentality of optimum detecting filter, enters the first input of correlators unit. Correlators unit along with PRS generator form the closed-loop system, which facilitates the

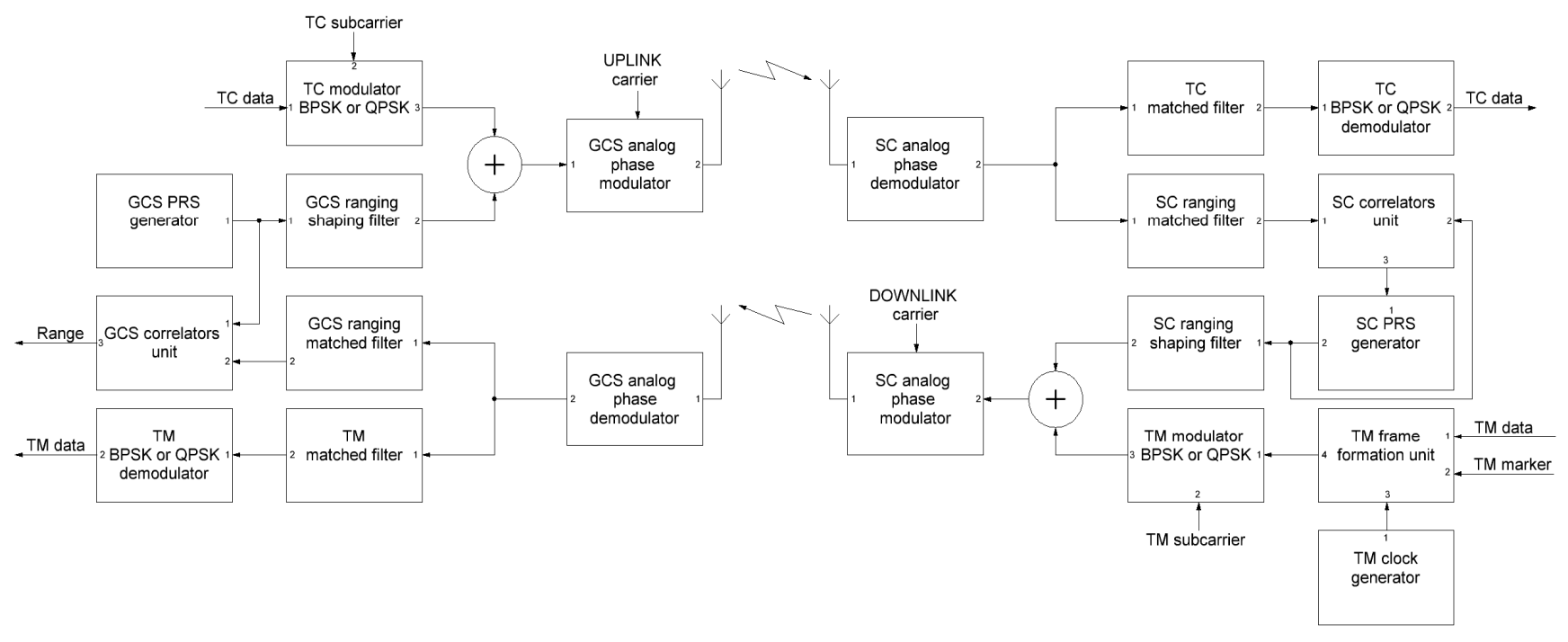

Fig. 1. SC CMS block diagram recommended by CCSDS standard 
synchronization of SC PRS generator signal with RANGING signal, received from earth. Signal from PRS generator output 2 enters the one of the summarizer inputs, TM signal on compliant subcarrier frequency enters the other input of the summarizer. Signal from the summarizer output, which is represented as sum of TM and RANGING signals, enters the first input of SC analog phase modulator, downlink carrier frequency enters the second input of phase modulator. Further wave modulated with carrier frequency enters the SC transmitting antenna, and then enters the GCS receiving antenna.

For modulation of TM signal subcarrier wave CCSDS standard recommends the application of BPSK or QPSK modulation, as it for TC signal modulation.

On earth signal from receiving antenna enters the GCS analog phase demodulator input 1. Signal from the demodulator output 2 enters inputs of RANGING and telemetry signals filters. TM signal, extracted by the means of matched filter, enters the first input of TM demodulator, telemetry data from the second output of demodulator are processed by GCS hardware. Range measurement signal, extracted by the instrumentality of optimum detecting filter, enters the first input of correlators unit. Signal from GCS PRS generator output 3 enters the input 2 of correlators unit. Correlators unit carries out the measurement of signal delay received from SC in relation to GCS PRS signal. Signal delay value is proportional to $\mathrm{SC}$ range.

In given SC CMS RANGING signal is represented as pseudorandom sequence (PRS), encrypted in that way that logic TRUE corresponds to $1 \mathrm{~V}$ signal level and logical FALSE corresponds to $-1 \mathrm{~V}$ signal level. Shaping filter is applied for RANGING signal spectrum limitations. Range measurement is conducted by means of computing the delay of RANGING signal transferring from GCS to SC and backwards and following conversion calculation of signal delay into range.

Feature of given diagram is application of complex twolevel modulation circuit [1]. At first level for TC, TM data subcarrier frequencies are formed, which are modulated by compliant data using discrete modulation, such as BPSK or QPSK. Modulated subcarriers enter the first input of adder. RANGING signal enters the second adder input. Adder output signal is transferred to GCS analog phase modulator input, where carrier frequency modulation is conducted. Spectrum of

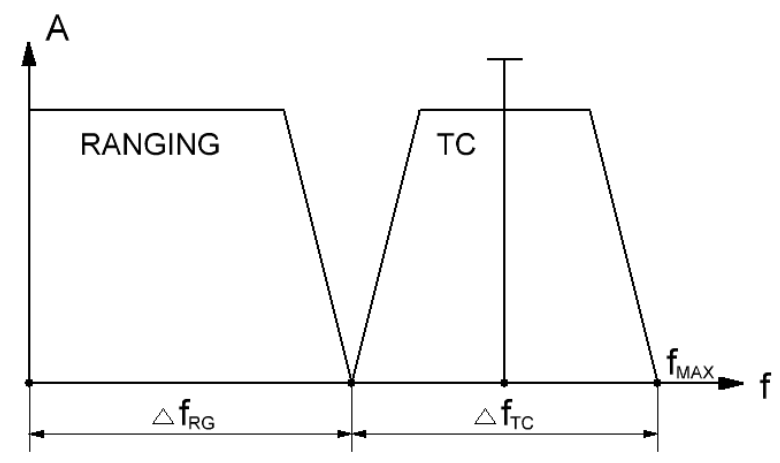

Fig. 2. Spectrum of signal at the analog phase demodulator input. signal at the analog phase modulator input is shown on fig. 2 .

Spectrum bandwidth of signal at the analog phase modulator input is calculated by formula:

$$
f_{M A X}=\Delta f_{R G}+\Delta f_{T C}
$$

where $\Delta f_{R G}-$ RANGING signal spectrum bandwidth, $\Delta f_{T C}$ - TC signal spectrum bandwidth.

Spectrum bandwidth of signal at the GCS analog phase modulator output:

$$
\Delta f_{M O D}=2\left(D_{P}+1\right) f_{M A X}=2\left(D_{p}+1\right)\left(\Delta f_{R G}+\Delta f_{T C}\right),
$$

where $D_{P}-$ phase deviation in radians.

As we see from fig. 2, application of two-level modulation circuit leads to decreasing of usage efficiency of frequency resources in comparison with simple discrete modulation. Analog phase modulation expands signal spectrum by factor of $2\left(D_{P}+1\right)$ [4].

For example, if phase deviation is 1 radian then signal spectrum expands by factor of 4. Usage of two-level modulation in GCS-SC and SC-GCS lines is conditional upon demand of CCSDS on providing continuous transmission of telemetry data during RANGING measurement. Herewith condition of continuous TC data transmission and RANGING measurement is not necessary [2].

In SC CMS, which diagram is shown on fig. 1, formation and sending of TM frame with specified time interval is executed automatically by signal provided by TM clock generator. As long as condition of continuous TC data transmission and RANGING measurement is not necessary as it said in CCSDS standard [1] it is possible to change scheme and perform sending of TM frame by special GCS-marker signal send by GCS-SC line. In that case time since marker signal is send till TM frame is received will be proportional to delay of signal propagation from GCS to SC and backwards, which means that it will be proportional to range from GCS to SC. In that case necessity of two-level modulation vanishes.

PRS of specified length can be applied in the function of GCS marker. Essential sequence length and data rate should be selected on the basis of needed accuracy and SC orbit parameters [5].

SC CMS, which scheme is shown on fig. 3, permits to perform TM data transmission and RANGING measurement simultaneously without two-level modulation application. 


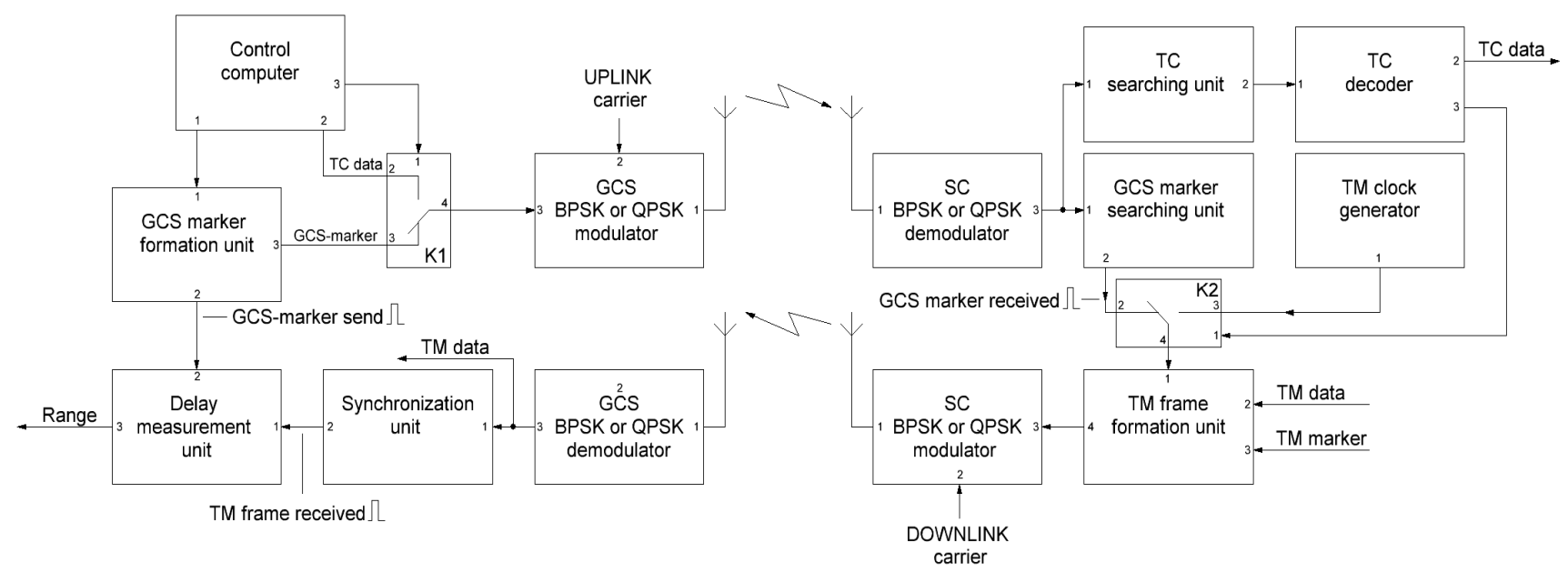

Fig. 3. SC CMS block diagram without application of two-level modulation

In provided on fig. $3 \mathrm{SC}$ CMS block diagram transmission line from GCS to SC is used for transferring two data types: TC and GCS-marker, which executes TM frame sending from $\mathrm{SC}$ to GCS. Selection of data type send on GCS-SC line is performed by operator with support of controller computer. In operating mode of TC information transmission controller computer executes communication of input 2 of $\mathrm{K} 1$ switch to computer's output 3 and performs TC data transmission to first input of GCS modulator through K1 switch.

In range measurement mode controller computer executes communication of input 3 of K1 switch to output 3 of GCSmarker formation unit. Also on command of computer marker formation unit generates GCS marker, which is transferred to first input of GCS modulator through K1 switch. Signal from the modulator output on uplink carrier frequency enters GCS antenna and further passes to SC receiving antenna.

On SC board signal from the receiving antenna output goes to first input of SC demodulator. Data from the demodulator output are processed by GCS-marker searching unit. In case if there was a transmission of TC from GCS data will appear on the TC searching unit output and further go to decoder.

In case if there was a transmission of GCS marker from earth in the moment of marker reception GCS marker searching unit will send «GCS marker received» signal to the output 2, which is further goes to input 1 of TM frame formation unit through $\mathrm{K} 2$ switch. By this signal's front TM frame formation unit performs $\mathrm{TM}$ frame transmission to the SC modulator input. In case if GCS marker transmission is impossible (for example if command line overloaded by big amount of transmitted command and program information) on command from earth $\mathrm{K} 2$ switch connects input 1 of TM frame formation unit to the output of TM clock generator and TM frames sending is performed by front of the signal from TM clock generator located on board of SC. From the modulator output signal on downlink carrier frequency enters the SC transmission antenna and further passes to the GCS receiving antenna.
From GCS receiving antenna signal goes to GCS demodulator input 1 . From the demodulator output 3 telemetry data are transferred to the synchronization unit input 1 . In the moment of complete reception of TM frame «TM frame received» signal is transferred to the synchronization unit input 2. Delay measurement unit carries out time measurement between fronts of «GCS marker send» and «TM frame received» signals.

$\mathrm{SC}$ range thus is evaluated by formula:

$$
R=C\left(T_{M E A S}-T_{A G C S}-T_{A S C}\right) / 2,
$$

where $\mathrm{T}_{\text {MEAS }}$ is time between fronts of «GCS marker send» and «TM frame received» signals, $T_{A G C S}$ and $T_{A S C}-$ hardware delays of the signal in GCS and SC line, which are measured on SC CMS test stage [5].

\section{CONCLUSION}

Reported in this article method of range measurement and command and measurement data transmission with application of simple types of modulation allows to increase frequency resource usage efficiency. Also absence of complex two-level modulation noticeably simplifies the circuitry of hardware, resulting in decreasing costs of SC CMS development and production.

Attempts of signal frequency bandwidth reduction under the conservation of SC CMS functionality were undertaken by developers earlier, but method described in this article differ in the fact that it allows to keep data transmission rates and noise immunity under sufficient economy of frequency resource. Analyses of system performance under the influence of different noises were not described in that article as long as they exceed the topic of this article. The issue of noise interference will be examined with more detail in further publications.

The reported study was funded by Krasnoyarsk Region Science and Technology Support Fund according to the research project: «Preparation for the introduction of the 
software and hardware complex for automation of tests of the on-board equipment of the tracking, telemetry and command subsystem in JSC "ISS"

\section{REFERENCES}

[1] CCSDS standard 401.0-B-20 (Radio Frequency and Modulation Systems - Part 1 Earth Stations and Spacecraft).
[2] ESA standard PSS-04-107 (European Space Agency Packet telecommand standard).

[3] С. П. Панько. Измерение дальности космического аппарата / С. П. Панько // Исследования наукограда. 2015 № 4, ноябрь-декабрь c. 10 .

[4] Н. М. Изюмов, Д. П. Линде. Основы радиотехники. М:, ЭНЕРГИЯ, 1965 г.

[5] А. С. Сыров. Бортовые системы управления космическими аппаратами. М:, МАИ-Принт, 2010. 